\title{
Analysis of Voltage Stability Index under Stressed Operating Conditions in Bus Power Systems
}

\author{
B. Vivekanadam \\ Professor, \\ Faculty of Computer Science and Multimedia, \\ Lincoln University College, Malaysia \\ vivekanandam@lincoln.edu.my
}

\begin{abstract}
In stressed operating conditions, several types of voltage stability indices (VSI) are used for the assessment of voltage stability at specific operating points. The performance of various available VSIs are compared in this paper. The one generation unit tripped effects, single line to ground (SLG) fault and inductive loading variations occur in combinational format with such operating conditions. Voltage collapse occurs in the lines or nodes due to the stressed operating conditions (SOC). SLG fault, loading effects, power margin, line continency ranking, and line number are some of the performance parameters of VSI analysed in this paper. For utilization of reactive power compensation, the proper location can be chosen with the help of critical line and node analysis (CLNA) that makes use of VSIs. For any SOC, accurate voltage instability prediction is performed using VSI as per the simulation results. Under voltage collapse due to multiple causes, the voltage stability assessment of any specific line can be performed using this information.
\end{abstract}

Keywords: Voltage stability indices, single line to ground fault, power margin analysis, one generation unit tripped, contingency analysis;

\section{Introduction}

The prevailing structure of electricity is pushed to operate under a burdened situation due to the new power lines and their excessive assembling cost, self-regulatory approaches of the power transmission and distribution industries, impact on nature, land use restrictions, landscape variation, climatic effect and other restrictions imposed by environmental parameters [1]. Voltage instability may occur if any perturbation is caused in such conditions. For any power system, voltage stability investigation is a vital parameter. The stable voltage points of a power system can be analysed using the static and dynamic voltage stability approach. When compared to the static approach, the dynamic stability analysis requires more computation time. Reactive power versus line voltage (QV) and real power versus line voltage (PV) are estimated for analysing the power system stability [2]. However, these methods are time-consuming. Quicker estimation of voltage stability can be performed using voltage stability indices (VSI) based techniques. The system's voltage stability is specified by the numerical value of VSI. Under general system conditions like multiple real load variation, single continuous reactive load variation, and base loading, various VSI models are proposed. The power system's severe stressed operating conditions are significant in choosing the right VSI [3].

Various performance analysis must be performed for evaluating the numeric value and appropriate VSI selection. For any specific node or line, under diverse stability analysis and stressed operating conditions, different VSI may be suitable [4]. In accordance to the system's voltage stability, critical lines, feeble buses in the system, maximum load acceptable and voltage collapse point may be predicted using the Fast Voltage Stability Index (FVSI) model. However, the line's real power flow is neglected leading to inaccuracy in the results when real power increment is faced by the FVSI model [5]. The reactive power is used for calculation of line stability index (LSI). When the line voltage is nearing its corresponding unstable point, lesser magnitude of LSI is obtained as the shunt admittance is almost zero since the real power flow is neglected.

Under different SOCs and loading conditions, the VSI of various lines are estimated in this paper. In various system loading conditions, the analysis of VSI in SOC is evaluated. Under diverse system loading conditions, CLNA for SOCs is performed separately [6]. For mixed load or reactive load and multiple real, reactive load and combination of pre-specified real and baseload, reactive loads and multiple real, reactive load and pre-specified real as well as base load, contingency analysis is performed. In case of single maximum load variation and single continuous load variation, the prediction of power margin (PM) by VSI is prolonged. PM prediction and accurate voltage stability prediction is estimated based on the most performing VSI on discussing all subcases [7].

\section{Literature Survey}

The reactive and real power is considered for estimation of line stability factor (LSI) to provide efficient results. The accuracy of results decline under heavy loading LSI as the resistance and admittance effects are not considered [4]. The voltage stability radial distribution is compensated with the LSI. As the reactive loading increases, 
Journal of Electrical Engineering and Automation (EEA) (2020)

Vol. 02/ No. 04

Pages: $146-150$

https://www.irojournals.com/iroeea

DOI: https://doi.org/10.36548/jeea.2020.4.001

inaccurate results are obtained by LSI as the reactive loading effects are neglected. The Novel Line Stability Index (NLSI) considers both reactive as well as real power loading effects. When compared to the VSI technique that does not consider the reactive and real power loading, the accuracy of results provided by NLSI is higher [8]. However, the line admittance value is neglected in the NLSI technique that leads to less accurate results in case of long distribution lines. Under varying load conditions, the accuracy of results reduces when implementing Voltage Collapse Proximity Indicator (VCPI) due to its constant power factor. The resistance of transmission line is neglected while considering the reactive and real load variation effects using the New Voltage Stability Index (NVSI) scheme [9]. The NVSI stability prediction accuracy is also less accurate as transmission line resistance is neglected in the long transmission lines.

The reactive power of the line is used as the key element in Voltage Reactive Power Index at Line (VRPIL). The VRPIL accuracy further reduces as the distance increases from the voltage collapse node. The Power Transfer Stability Index (PTSI) is implemented in power systems for predicting the dynamic voltage collapse [3]. However, calculation of PTSI is performed under zero admittance of line. In voltage stability prediction, the accuracy may be inconsistent in case of large line admittance as zero admittance is considered while calculating PTSI [10]. Power margin dependant online VSI is proposed by certain researchers. During voltage collapse point prediction, the accuracy is low as constant power load is considered and power margin is not predicted accurately. The phasor measurement units (PMU) that are located optimally provide data for estimation of Voltage Stability Load Index (VSLI). The optimal placement can be performed using different techniques for PMUs that are responsible for VLSI [11]. The IEEE-14 bus system has the greatest impact on voltage stability when compared to the IEEE-33 system as it has the maximum number of generators and forms the ring main power system. The IEEE-14 bus system is considered in most literature. Hence, the IEEE-14 bus system is considered as a standard in this paper. When compared to normal operating conditions, the probability of simultaneous occurrence of practical power system disturbances are termed as SOCs. Also, among all operating conditions, the chances of voltage instability occurrence is high in SOCs [12].

\section{Proposed Work}

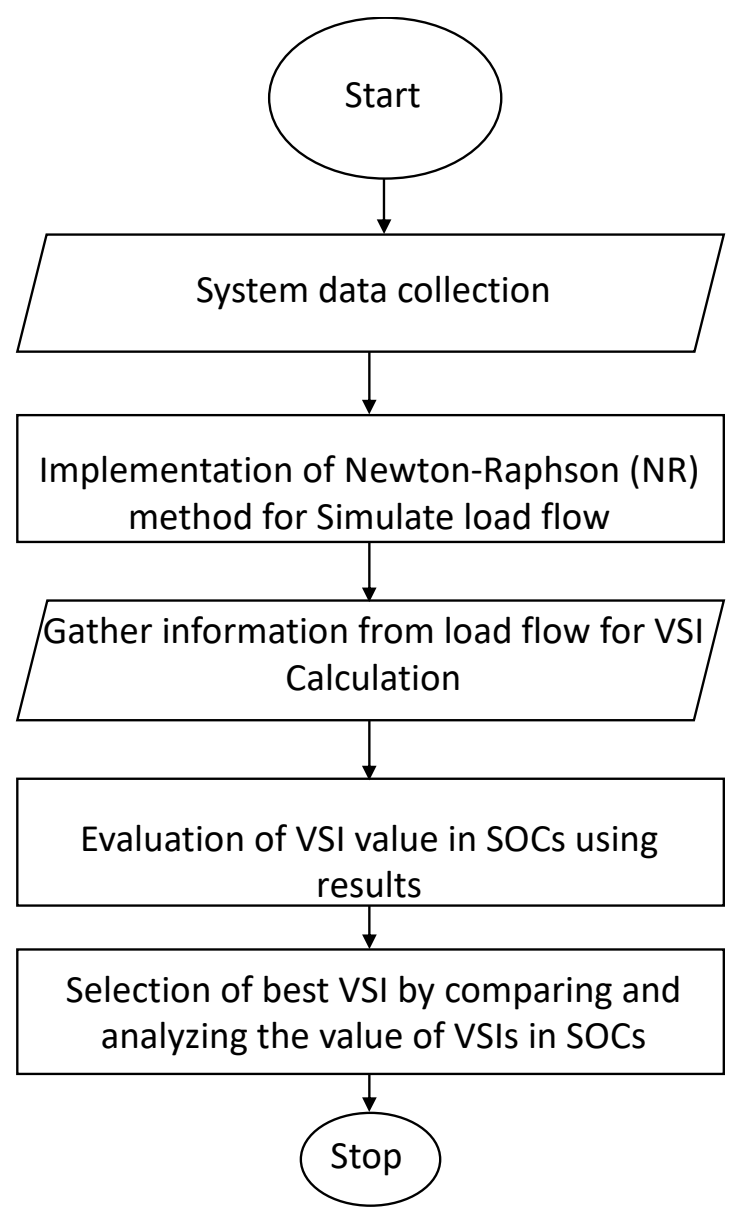

Figure 1: Flow chart of the proposed model 
Journal of Electrical Engineering and Automation (EEA) (2020)

Vol. 02/ No. 04

Pages: 146-150

https://www.irojournals.com/iroeea

DOI: https://doi.org/10.36548/jeea.2020.4.001

Several operating conditions like SOCs, varying load and baseload are used for computing VSI in IEEE14 bus power system. Figure 1 represents the flow chart of the steps involved in VSI calculation under diverse loading conditions. IEEE 14 bus standard datasheet is used for obtaining transformer parameter, reactive and load real power, reactive and generator real power, reactance and resistance of line and other system data. Standard parameter values for load, transformers, generators and transmission lines are used for developing the Simulink model for IEEE 14 bus in MATLAB environment. Newton-Raphson method is used for conducting load flow analysis of the simulated IEEE bus system. On successive simulation, load flow report provides system data inclusive of power angle, end reactive power for transmitting and receiving end, active power for transmitting and receiving end, voltages of transmitting and receiving end and so on. Further, with respect to the system data derived from the load flow, evaluation of VSIs for different busses are performed. Prediction of required voltampere reactive (VAR) compensation location and amount, Mega Volt Ampere (MVA) margin and line or bus stability condition is performed with the help of VSI magnitude. Finally, appropriate VSI is selected for the specific case on comparison of the VSIs in terms of advantages, disadvantages and assessment schemes.

\section{Results and Discussion}

In IEEE 14 bus system, bus one is identified as the slack bus and buses two, four, six and eight are the PV buses. Rest of the buses are load buses. Further, twenty interconnected lines are available in the system. The data gathered from load flow is used for calculation of different VSI. This section provides the test results of various stressed operating conditions. The VSI is obtained for general loading conditions inclusive of reactive and multiple real, only multiple reactive, only multiple real, reactive and single maximum real, single maximum reactive only, single maximum real only, reactive and single continuous real, only single continuous reactive, only single continuous real load change as well as base loading. The SOCs are simulated and compared for all the VSIs. Selective SOCs are deliberated in the following section. Based on reactive and active power margin and voltage magnitude, the most critical line is decided for selecting the suitable VSI in any specific case. Based on the data acquired by the load flow analysis, the line consisting of lower power margin and voltage magnitude is identified. In any specific SOC, the most performing VSI is selected on identifying the most critical line.

When compared to the underground power dispatch, the fault occurrence probability is more in overhead electric power transmission and distribution. Three-line to the ground, line to line, double line to the ground and single line to ground (SLG) faults are some of the fault types. SLG fault is considered as the probability of occurrence of this fault is high. The conditions under which SLG faults are studied includes reactive and single maximum real loading with SLG fault in multiple lines one at a time, single maximum reactive loading with SLG fault in multiple lines one at a time, single maximum real loading with SLG fault in multiple lines one at a time and base case loading with SLG fault in multiple lines one at a time. In terms of voltage instability, reactive and single maximum real loading with SLG fault in multiple lines one at a time is considered to be most critical. Figure 2 represents the performance analysis of VSI under this case. The most appropriate VSI may be followed for other cases.

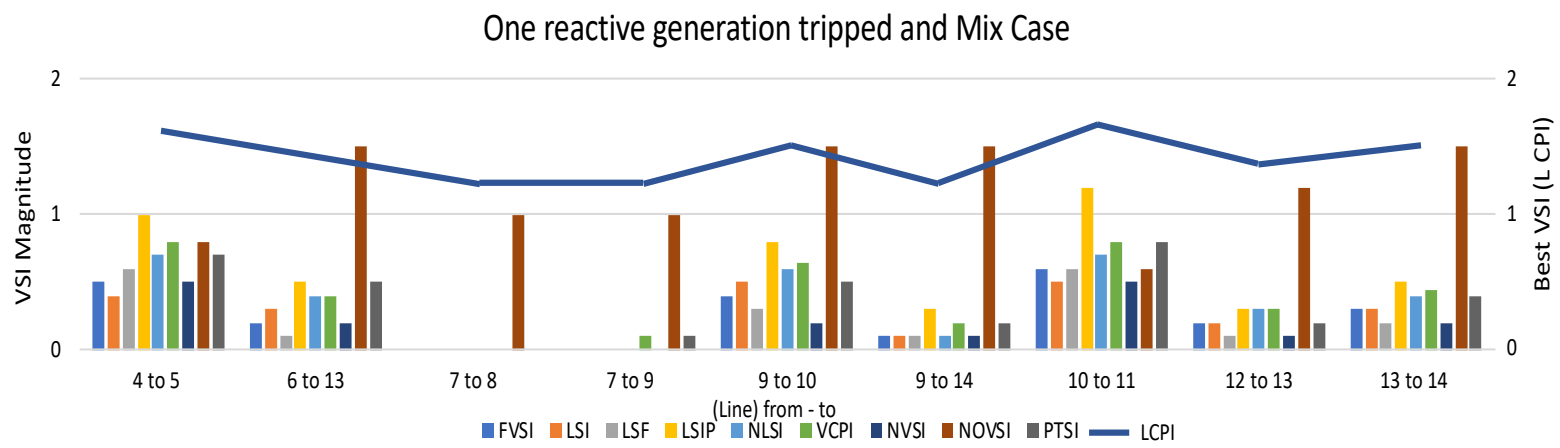

Figure 2: Single reactive power generation unit tripped and mixed loading case for general VSI representation

Figure 2 provides the comparison of different VSIs. The VSI found on LCPI is considered best due to its magnitude. Further, similar approaches are compared with this analysis along with heavy reactive loading, base case loading and the case in which the reactive power support is turned off. 


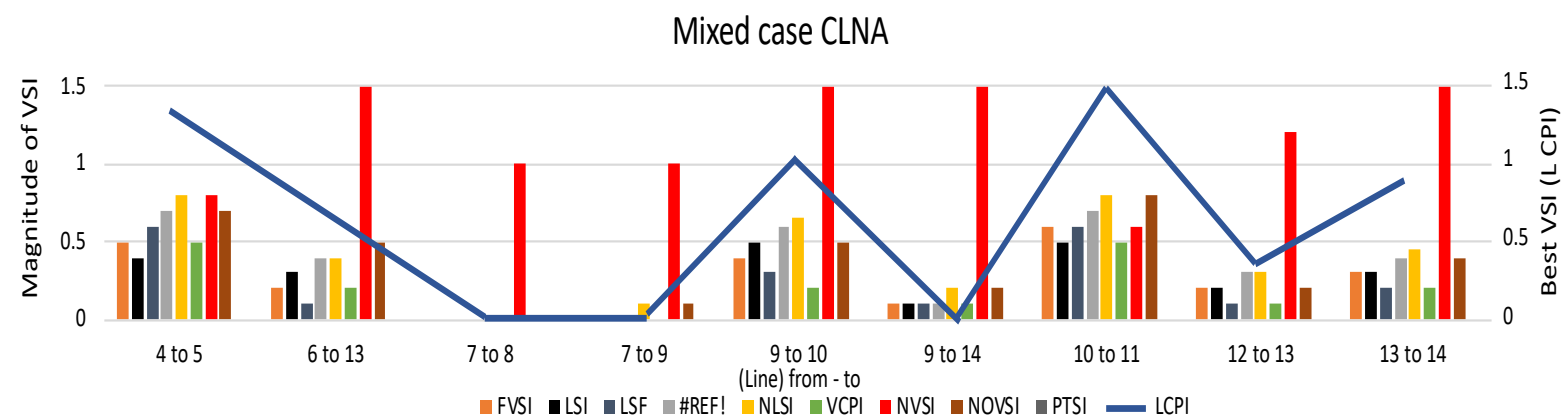

Figure 3: Mixed loading case with CLNA in VSIs

For multiple lines, the variation of magnitude of VSI is represented in figure 3. The VSIs are compared for multiple lines with mixed loading cases and the CLNA is analysed. Further, the variation and dominance of Line Collapse Proximity Index (LCPI) is also validated by the simulated results.

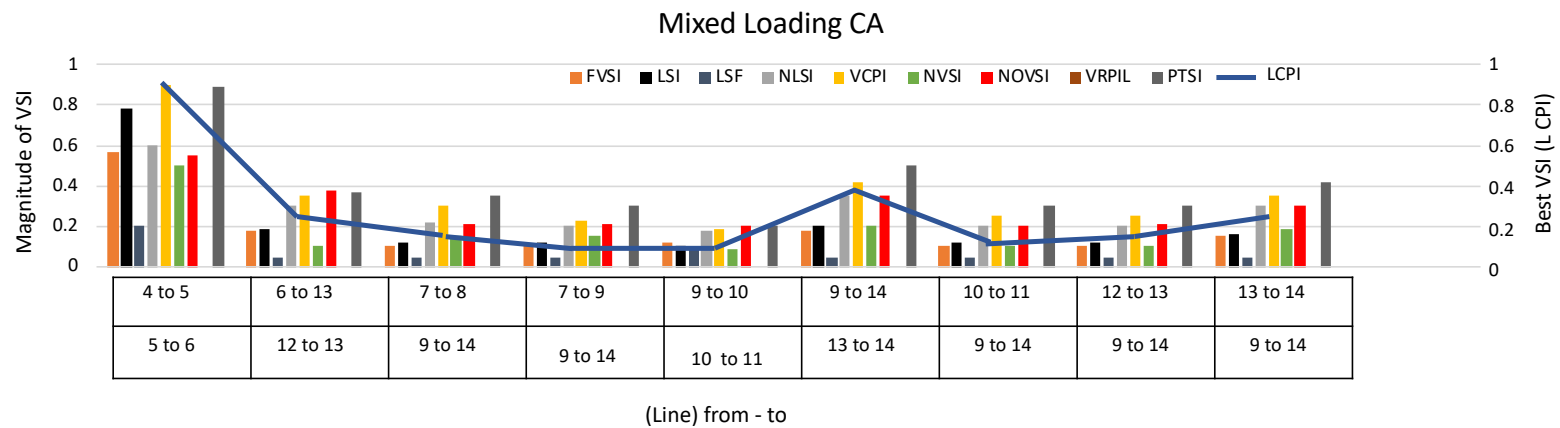

Figure 4: Mixed loading case with CA in VSIs

At nodes 14, 13, 12, 11, 10, 9, 5 and 4 the loading multiplier is considered along with pre-specified loads in mixed loading case. Figure 4 represents multiple line VSI variation. The LCPI and best VSI are depicted graphically.

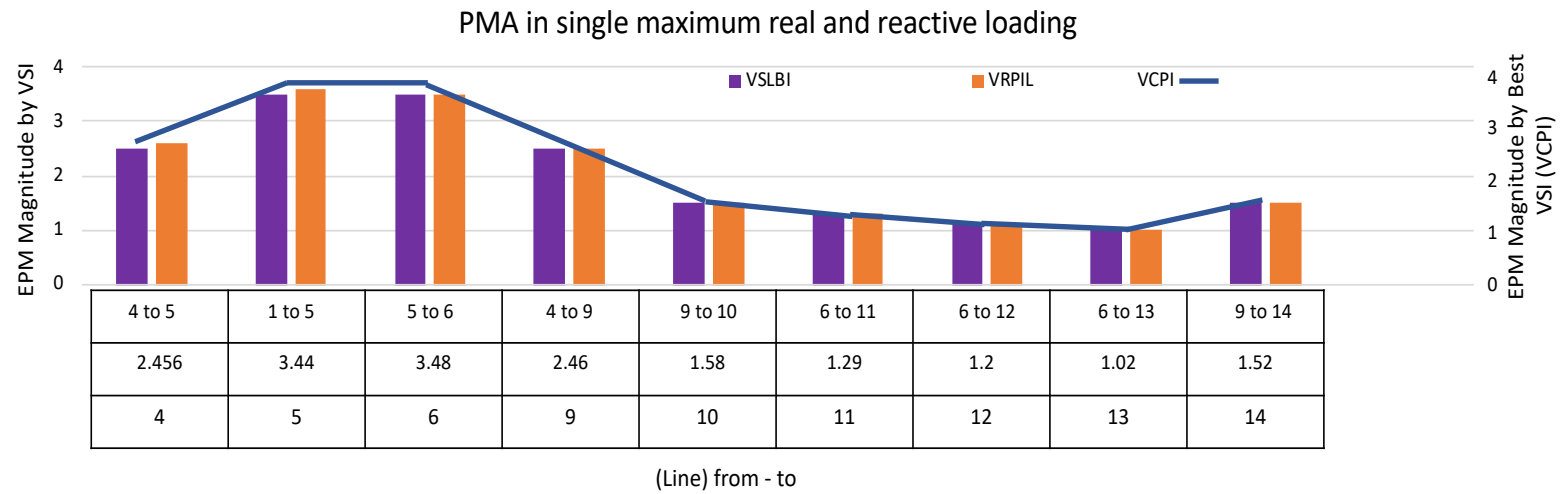

Figure 5: Reactive and single continuous loading with PMS in VSIs

The evaluated power margin and for actual power margin are represented in figure 5. For multiple lines, the VSI magnitudes are represented with power margin variations. The best VSI for the case under study is depicted in the secondary axis.

\section{Conclusion}

Various lines and their stressed operating conditions are examined in an IEEE 14 bus system along with the VSI estimation in this paper. Mixed loading case, power generation unit tripped one reactive case, single line to ground fault, reactive load and pre-specified real change, multiple load change, single maximum load change, 
Journal of Electrical Engineering and Automation (EEA) (2020)

Vol. 02/ No. 04

Pages: $146-150$

https://www.irojournals.com/iroeea

DOI: https://doi.org/10.36548/jeea.2020.4.001

single continuous load change and base case loading are analysed. When the buses or lines face specific stressed operating conditions, the performance is analysed for selection of appropriate VSI with the help of system conditions. Accurate prediction of power margin lines, contingency analysis, node and critical line analysis, effect of a single reactive support going off on VSI, effect of ground fault to single line on VSIs, VSI loading effects and VSI magnitude are some of the VSI performance parameters considered in this paper. During the system condition variations, the voltage instability of lines can be detected effectively and accurately based on the information acquired from the VSI as represented in the graphical outputs. On multiple lines, for any specific case, the competence of a specific VSI is represented by the results. Other cases like best performing VSI variations, One generation unit tripped (OGT), and Single line to ground (SLG) fault are analysed. Future work is directed towards analysis of more performance parameters and improving the efficiency of selected VSI.

\section{References}

[1] Ratra, S., Tiwari, R., \& Niazi, K. R. (2018). Voltage stability assessment in power systems using line voltage stability index. Computers \& Electrical Engineering, 70, 199-211.

[2] Chandra, A., \& Pradhan, A. K. (2019). Online voltage stability and load margin assessment using wide area measurements. International Journal of Electrical Power \& Energy Systems, 108, 392-401.

[3] Rodriguez-Garcia, L., Perez-Londono, S., \& Mora-Florez, J. (2019). An optimization-based approach for load modelling dependent voltage stability analysis. Electric Power Systems Research, 177, 105960.

[4] Tamta, R., Painuli, S., Rawat, M. S., \& Vadhera, S. (2018, January). Comparison of line voltage stability indices for assessment of voltage instability in high voltage network. In 1st International Conference on New Frontiers in Engineering, Science \& Technology (NFEST-2018) (pp. 8-12).

[5] Burle, T., Chintapalli, V. B. R., \& Thota, P. (2020). A new line voltage stability index (NLVSI) for voltage stability assessment. In Intelligent computing techniques for smart energy systems (pp. 535-548). Springer, Singapore.

[6] Nageswa Rao, A. R., Vijaya, P., \& Kowsalya, M. (2018). Voltage stability indices for stability assessment: a review. International Journal of Ambient Energy, 1-17.

[7] MEENA, M. K. (2019). PREDICTION AND ENHANCEMENT OF VOLTAGE STABILITY OF POWER SYSTEMS (Doctoral dissertation).

[8] Kamel, M., Karrar, A. A., \& Eltom, A. H. (2017). Development and application of a new voltage stability index for on-line monitoring and shedding. IEEE Transactions on power systems, 33(2), 1231-1241.

[9] Venkateswarlu, S., \& Kishore, T. S. (2021). Enhancement of Static Voltage Stability Margin Using STATCOM in Grid-Connected Solar Farms. In Control Applications in Modern Power System (pp. 387398). Springer, Singapore.

[10]Bhalaji, N. (2020). EL DAPP-An Electrıcity Meter Trackıng Decentralızed Application. Journal of Electronics, 2(01), 49-71.

[11] Ranganathan, G., \& Smys, S. Smart Wireless sensors for Impairment detection of the offshore Wind Turbines.

[12] Kumar, A. D. Flawless Attuning for Parameters of Power System Modulator Applying Grey Wolf Optimization. 